\title{
Novel broadband microstrip- to-CPW transition with easy transmission band control
}

\author{
Jung-Woo Baik ${ }^{\text {a) }}$, Tae-Hak Lee, and Young-Sik Kim \\ Dept. of Radio Sciences and Engineering, Korea University \\ 5-ga, Anam-dong Sungbuk-gu, Seoul 136-713, Korea \\ a)baik-jw@korea.ac.kr
}

\begin{abstract}
A broadband microstrip-to-coplanar waveguide (CPW) transition using two parallel folded balun structures with easy transmission band control for various RF circuits and integrated systems is proposed in this paper. Each balun is employed an open-circuited microstrip line and a short-circuited slotline with one quarter-wavelength long each. As the change of the length of the open and short circuits, the transmission band can be easily controlled. The measured return and insertion losses are better than $10 \mathrm{~dB}$ and $1.4 \mathrm{~dB}$, respectively, over the bandwidth of $82.5 \%$ at the center frequency of $6 \mathrm{GHz}$.
\end{abstract}

Keywords: microstrip line, coplanar waveguide, transition structure, broadband balun

Classification: Microwave and millimeter wave devices, circuits, and systems

\section{References}

[1] R. N. Simons, Coplanar waveguide circuits, components, and systems, New York: John Wiley \& Sons, 2001.

[2] T. J. Ellis, J.-P. Raskin, L. P. B. Katehi, and G. M. Rebeiz, "A wideband CPW-to-microstrip transition for millimeter-wave packaging," IEEE MTT-S Int. Microw. Symp. Dig., vol. 2, pp. 629-632, June 1999.

[3] T.-N. Kuo, S.-C. Lin, and C. H. Chen, "Compact Ultra-wideband bandpass filters using composite microstrip-coplanar waveguide structure," IEEE Trans. Microw. Theory Tech., vol. 54, no. 10, pp. 3772-3778, Oct. 2006.

[4] J.-C. Chiu, J.-M. Lin, M.-P. Houng, and Y.-H. Wang, "A PCBcompatible 3-dB coupler using microstrip-to-CPW via-hole transitions," IEEE Microw. Wirel. Compon. Lett., vol. 16, no. 6, pp. 369-371, June 2006.

[5] R. N. Simons and R. Q. Lee, "Coplanar-waveguide/microstrip probe coupler and applications to antennas," Electron. Lett., vol. 26, no. 24, pp. 1998-2000, Nov. 1990.

[6] L. Zhu and W. Menzel, "Broad-band microstrip-to-CPW transition via frequency-dependent electromagnetic coupling," IEEE Trans. Microw. Theory Tech., vol. 52, no. 5, pp. 1517-1522, May 2004.

[7] N. I. Dib, R. N. Simons, and L. P. B. Katehi, "New uniplanar transitions for circuit and antenna applications," IEEE Trans. Microw. Theory Tech., vol. 43, no. 12, pp. 2868-2873, Dec. 1995. 
[8] B. Edward and D. Rees, "A broadband printed dipole with integrated balun," Microw. J., vol. 30, no. 5, pp. 339-344, May 1987.

\section{Introduction}

The microstrip-to-coplanar waveguide $(\mathrm{CPW})$ transitions with broadband and low insertion loss have been developed due to arousing interest in hybrid or multilayered microwave integrated circuits (MICs) as well as in monolithic microwave integrated circuits (MMICs) $[1,2]$. Also, the microstrip-to$\mathrm{CPW}$ transitions are very useful in RF devices such as couplers and filters $[3,4]$. Several techniques for microstrip-to-CPW transitions have been reported such as a via-hole and a metal post based on electrical contact [5], an electromagnetic surface-to-surface coupling [6], and a phase shifting technique [7]. Although the aforementioned transitions have broadband characteristic, those transitions are involved the difficulty of transmission band control for various RF circuits and integrated systems.

In this paper, a new microstrip-to-CPW transition using two parallel folded balun structures is proposed with broadband characteristic. This new transition is easy to control a transmission band by simple circuit parameter change. The folded balun used in this design has been initially reported for producing a balanced mode, such as a coplanar stripline (CPS) mode [8]. The simulation and optimization have been carried out using the commercial software IE3D based on the method of moment. Details of the transition design and experimental results are presented.

\section{Transition Design}

The proposed transition is fabricated on a RT/Duroid 5880 substrate with a dielectric constant of 2.2 for broadband and a thickness of $0.254 \mathrm{~mm}$ for tight coupling. Figure 1 (a) shows the layout of the proposed transition with two parallel folded balun structures. Its equivalent circuit is shown in Fig. 1(b).

Each balun consists of the short-circuited slotline of the CPW beneath the open-circuited microstrip line. The characteristic impedance of $50 \Omega$ at each port can be obtained by selecting proper values of $w_{1}=0.76 \mathrm{~mm}$, $w_{2}=3.4 \mathrm{~mm}$, and $s=0.1 \mathrm{~mm}$. The width of the split microstrip line with $100 \Omega$ is $w_{3}=0.22 \mathrm{~mm}$.

In this paper, the folded balun consisting of open/short circuits with one quarter-wavelength long is employed for field and impedance matching simultaneously between the $100 \Omega$ microstrip line and the characteristic impedance of the CPW slotline which represents $Z_{a b}$. Because the points of 'A' and 'B' as shown in Fig. 1 (b) are virtually shorted by the open-circuited microstrip line with one quarter-wavelength long, the field matching between a microstrip mode and a CPW mode can be achieved. And the impedance matching can be easily obtained by removing the effect of the open/short circuits in $Z_{i n}^{\prime}$. 


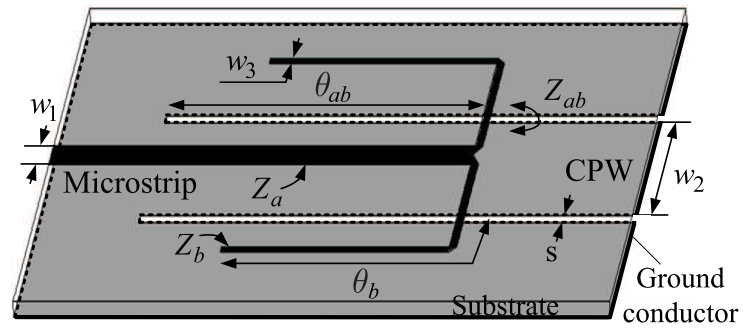

(a)

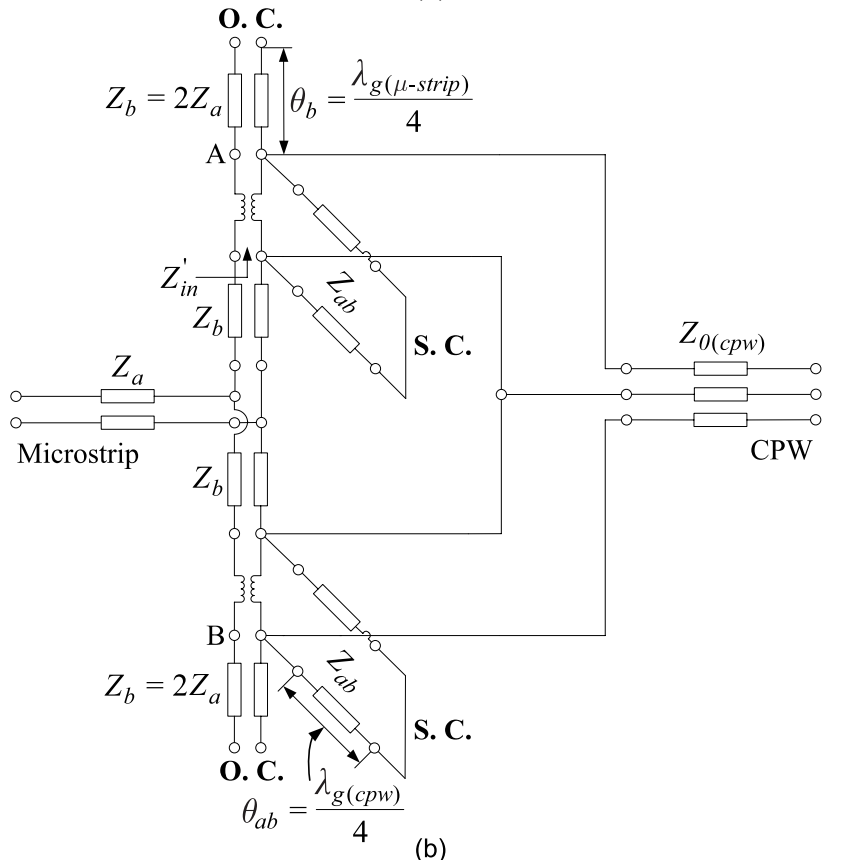

Fig. 1. The proposed transition with two parallel folded balun structures

The input impedance $Z_{\text {in }}^{\prime}$ shown in Fig. 1 (b) can be expressed by [8]

$$
Z_{i n}^{\prime}=-j Z_{b} \cot \theta_{b}+\frac{j 2 Z_{0(c p w)} Z_{a b} \tan \theta_{a b}}{2 Z_{0(c p w)}+j Z_{a b} \tan \theta_{a b}},
$$

Where $Z_{0(c p w)}$ is the characteristic impedance of the CPW, that is $50 \Omega$, and $Z_{b}$ and $\theta_{b}$ are the characteristic impedance and the electrical length of the open-circuited microstrip line, respectively. $Z_{a b}$ is the characteristic impedance between the central conductor and the one sided ground conductor. $\theta_{a b}$ is the electrical length of the short-circuited CPW. Each one quarter-wavelength $\left(\theta_{a b}=\theta_{b}=\pi / 2\right.$ in Fig. $\left.1(\mathrm{~b})\right)$ of the open-circuited microstrip line and the short-circuited $\mathrm{CPW}$ is utilized at the center frequency. Thus, the input impedance in equation (1) can be reduced as follows:

$$
Z_{\text {in }}^{\prime}=2 Z_{0(\mathrm{cpw})} \text {. }
$$

In this design, because $Z_{0(\text { cpw })}$ is chosen to $50 \Omega$, the impedance matching between the $100 \Omega$ microstrip line and the slotline of the CPW can be easily achieved at $6 \mathrm{GHz}$. 


\section{Results}

Figures 2 (a) and 2 (b) are the simulated insertion and return losses of the proposed transition for three different center frequencies, respectively. Electrical lengths of open/short circuits are one quarter-wavelength at each center frequency. It can be seen that the transmission band is changed while still keeping $3 \mathrm{~dB}$ insertion loss bandwidth of $114 \%$ in three cases.

Figure 3 is the measured and simulated frequency responses when all of the electrical length of $\theta_{a b}$ and $\theta_{b}$ are $\pi / 2$ at the center frequency of $6 \mathrm{GHz}$. The physical lengths of $\theta_{a b}$ and $\theta_{b}$ are $11 \mathrm{~mm}$ and $9.5 \mathrm{~mm}$, respectively. The measured insertion loss of the transition is less than $1.4 \mathrm{~dB}$ over the frequency range of 3.53 to $8.48 \mathrm{GHz}$, which includes the loss of two SMA connectors. The measured return loss is lower than $10 \mathrm{~dB}$ over the same frequency band.

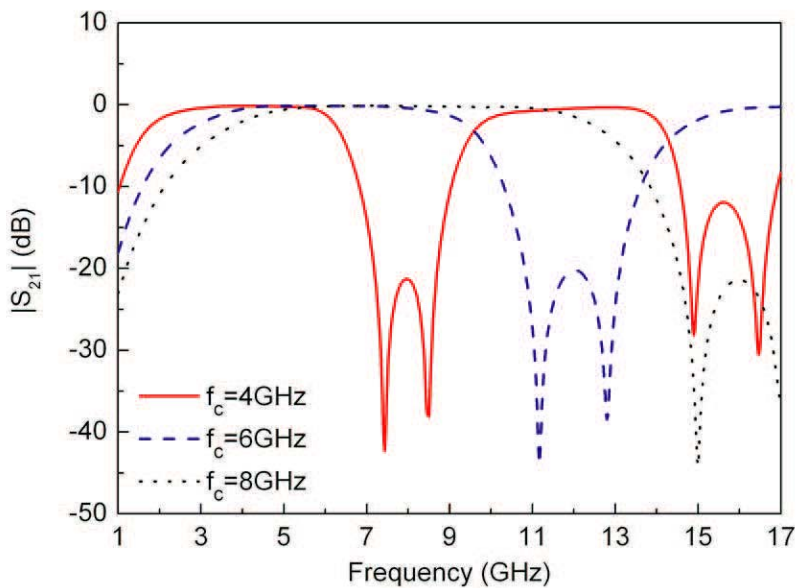

(a)

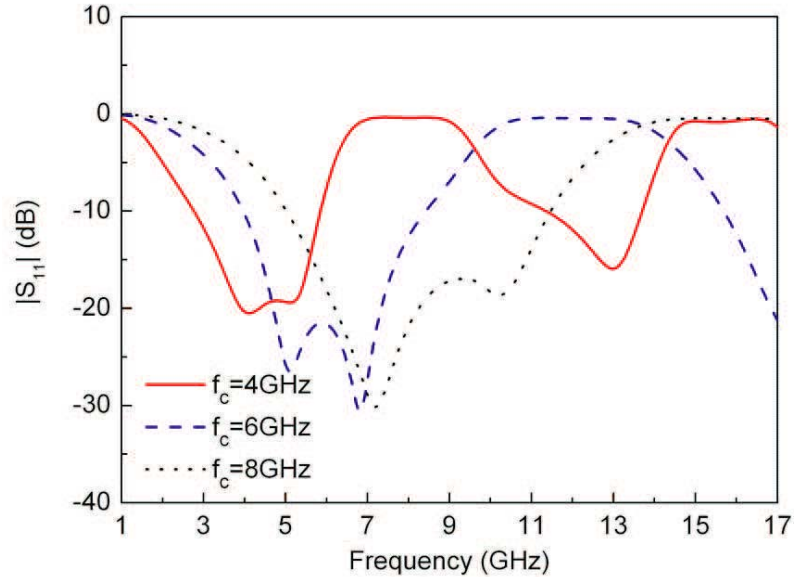

(b)

Fig. 2. The simulated frequency responses related to three center frequencies

\section{Conclusion}

A broadband microstrip-to-CPW transition is proposed using two parallel balun structures and analyzed in this paper. Field and impedance matching 


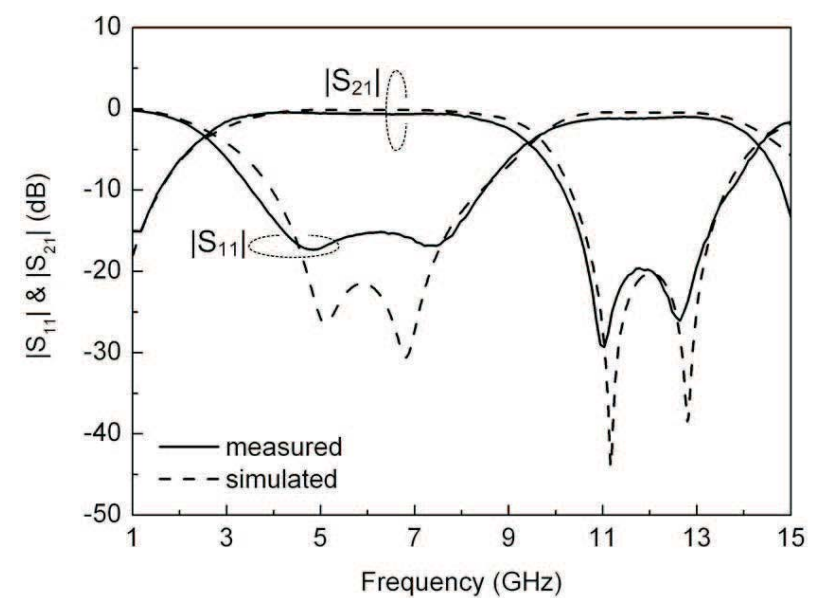

Fig. 3. The measured and simulated frequency responses

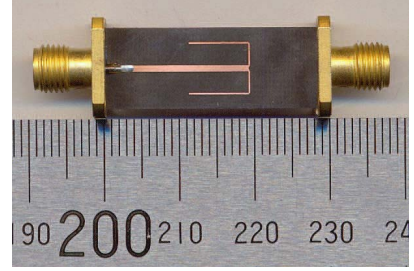

(a)

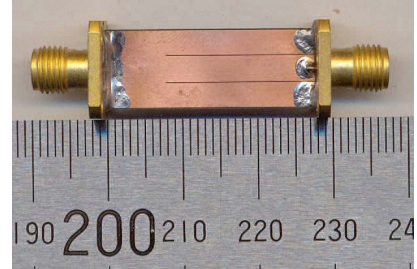

(b)

Fig. 4. Photographs of a fabricated broadband transition with balun structure: (a) Top view. (b) Bottom view.

can be easily achieved by means of balun structure from an open-circuited microstrip line and a short-circuited CPW.

The measured insertion and return losses are better than $1.4 \mathrm{~dB}$ and $10 \mathrm{~dB}$, respectively, from 3.53 to $8.48 \mathrm{GHz}(82.5 \%$ bandwidth at $6 \mathrm{GHz})$. Because of the easy transmission band control, this transition can be applicable to various broadband RF circuits and integrated systems. 